\title{
Comparison of Intensity Modulated Radiation Therapy (IMRT) and Three Dimensional Conformal Radiotherapy (3DCRT) In Supratentorial Astrocytic Series WHO Grade III-IV Primary Malignant Brain Tumors
} Khaleel IA ${ }^{1}$, Govardhan HB $^{1 *}$ and Senthil kumar ${ }^{2}$

${ }^{1}$ Department of Radiation Oncology, Kidwai Memorial Institute Oncology, Bangalore, Karnataka, India

${ }^{2}$ Department of Medical physics, Kidwai Memorial Institute Oncology, Bangalore, Karnataka, India

*Corresponding author: Govardhan HB, Department of Radiation Oncology, Kidwai Memorial Institute Oncology, Bangalore, Karnataka, India, E-mail: govardhanhb@gmail.com

Received date: July 19, 2018; Accepted date: August 14, 2018; Published date: August 24, 2018

Copyright: $\odot 2018$ Khaleel IA, et al. This is an open-access article distributed under the terms of the Creative Commons Attribution License, which permits unrestricted use, distribution, and reproduction in any medium, provided the original author and source are credited.

\begin{abstract}
Objectives: To determine whether Intensity Modulated Radiotherapy (IMRT) improves target coverage, target homogeneity, target conformity, critical tissue sparing without increasing the total integral dose to the non-target brain tissue to the three dimensional conformal radiotherapy (3DCRT) in Supratentorial Astrocytic series WHO grade III-IV primary malignant brain tumor.
\end{abstract}

Methods and Materials: Sixty patients of malignant glioma treated with 3DCRT were selected for a comparative dosimetric evaluation with IMRT. Target volumes, organ at risk (OAR), dose volume constrains were used for planning. Cumulative dose volume histogram of target volumes and organ at risk (OAR), normal brain tissue integral dose, target coverage, target homogeneity, target conformity, and normal tissue sparing with 3DCRT and IMRT planning were compared. Statistical analysis was performed to determine the differences and significance.

Results: In all 60 patients examined, there was no significant difference in target coverage between IMRT and 3DCRT plans with slightly superiority in 3DCRT plan in the range of $95 \%-100 \%$ of prescribed. IMRT improved target conformity, dose reduction to normal tissues including brain stem ( $D_{\text {mean }}$ by $17 \%, D_{\max }$ by $\left.12 \%\right)$, optic chiasm $\left(D_{\text {mean }}\right.$ by $33 \%, D_{\max }$ by $\left.22 \%\right)$, Rt optic nerve $\left(D_{\text {mean }}\right.$ by $54 \%, D_{\max }$ by $\left.46 \%\right)$, Lt optic nerve $\left(D_{\text {mean }}\right.$ by $42 \%$, $D_{\max }$ by $\left.38 \%\right)$, Rt eye $\left(D_{\text {mean }}\right.$ by $37 \%, D_{\max }$ by $\left.29 \%\right)$, Lt eye $\left(D_{\text {mean }}\right.$ by $33 \%, D_{\max }$ by $\left.35 \%\right), p<0.001$. This was achieved without increasing total non-target brain tissue integral dose. Overall integral dose to brain tissue reduced by approximately $8 \%, p<0.001$ with IMRT compared with 3DCRT.

Conclusion: These results indicate that IMRT treatment for high grade glioma allows for improved target conformity, better critical tissue sparing without increasing the integral dose to normal brain tissue.

Keywords: 3DCRT; IMRT; Supratentorial astrocytoma; High grade glioma

\section{Introduction}

Despite optimal treatment with chemo radiation and adjuvant chemotherapy, the median survival is only 12 to 15 months for patients with glioblastoma (WHO grade IV) and 2 to 5 years for patients with anaplastic astrocytoma (WHO grade III). Three-dimensional conformal radiation therapy (3DCRT) was developed more than three decades ago with aim is to increase tumor control probability by improving the spatial distribution of dose relative to that achievable with traditional techniques. This could be accomplished by reducing normal tissue irradiation and consequently increasing the prescribed dose until normal tissue tolerance is reached. However, it has been argued that increased tumor dose may not be sufficient to increase local control due to the inherent radio resistance of some tumors and patient population heterogeneity [1].

IMRT is a more advanced form of three-dimensional conformal radiotherapy (3D-CRT) that relies on advanced linear accelerator with multileaf collimator technology to deliver several non-uniform intensity modulated beams, leading to increased dose-target conformity, the resultant isodoses are highly conformal with superior target coverage and reducing radiation dose to radiosensitive normal tissues close to the tumor even if they lie within a concavity of the target volume, compared to other external beam photon radiation techniques. Planning and delivery are resource intensive and require specific and costly software and hardware [2-4].

Complex dose distributions can be delivered that avoid a number of radiosensitive normal tissues close to a tumor. The appropriateness of these two approaches is likely to depend on the tolerance doses of surrounding radiosensitive normal tissues. This approach has been demonstrated to improve tumor coverage while decreasing the dose to critical structures in the head and neck and other sites. Utility of these IMRT techniques in malignant glioma has not yet been fully elucidated. At present there is very little dosimetric data regarding the dose distribution from IMRT in the treatment of malignant gliomas [3-7]. Thus main aim of our study is to determine whether Intensity Modulated Radiotherapy (IMRT) improves target coverage, target homogeneity, target conformity, critical tissue sparing without increasing the total integral dose to the non-target brain tissue in comparison to the three dimensional conformal radiotherapy in 
Citation: Khaleel IA, Govardhan HB, Senthil K (2018) Comparison of Intensity Modulated Radiation Therapy (IMRT) and Three Dimensional Conformal Radiotherapy (3DCRT) In Supratentorial Astrocytic Series WHO Grade III-IV Primary Malignant Brain Tumors. J Nucl Med Radiat Ther 9: 368. doi:10.4172/2155-9619.1000368

Page 2 of 7

Supratentorial Astrocytic series WHO grade III-IV primary malignant brain tumor

\section{Materials and Methods}

A total of 60 post-operative, histologically proved Supratentorial Astrocytic series WHO grade III-IV primary malignant tumors fulfilling eligibility criteria registered in the Department of Radiotherapy at Kidwai memorial institute of oncology, Bangalore were taken up for the study. Institutional scientific review board and ethical review board approval was obtained. The main inclusive criteria were histologically proven supratentorial Astrocytic series WHO grade III-IV primary malignant brain tumor either gross total resection/ subtotal resection/ biopsy with Karnofsky Performance Status scale $>60$, were samples of this study. The exclusive criteria were patient with metastatic brain tumor, patient with infratentorial brain tumor, patient received prior radiotherapy, patient with Karnofsky Performance Status scale $<60$ were excluded from the study.

\section{Immobilization of the patient}

Individually designed thermoplastic mold immobilization devices are produced in neutral head and neck position for each patient, to better ensure positioning can be performed in a reproducible fashion for both planning and treatment. The mold is custom fit to the patient and then clamped to the treatment couch.

\section{Initial simulation}

The patient is positioned in the CT scanner on a flat table top with the immobilization cast. The patient was positioned using the reference localization marks and the CT alignment lasers. Radio-opaque fiducials are placed on the localization marks to identify the provisional isocenter location on the $\mathrm{CT}$ scans. Anterior and lateral radiographic topogram views were acquired to confirm the isocenter position and also the alignment of the patient's bony anatomy.

\section{D imaging}

The patient should be on empty stomach, history of any drug allergy should be enquired prior to examination. Ultravist contrast material of $100 \mathrm{ml}$ is $(1.5 \mathrm{ml} / \mathrm{kg})$ infused; CT images are taken as $3 \mathrm{~mm}$ axial slices from vertex up to $\mathrm{C} 2$ vertebral level. These CT images are transferred to the Varian eclipse treatment planning system (TPS) and images were later registered in the TPS. Post-operative T1W contrast MRI axial images of $3 \mathrm{~mm}$ slices taken within 1 week after surgery or T2W MRI images of biopsy only patients is co registered in the same. The result was superimposed on the patient's CT scan. Note that the particular target is clearly seen on the MR image but only portions of it are observed on the CT scan.

\section{Initial target volume}

Target volumes delineation will be based upon postoperativeenhanced MRI. The initial gross tumor volume (GTV1) will be defined by either the T2 or the FLAIR abnormality on the postoperative MRI scan. This must also include all postoperative-enhanced MRI regions and the surgical cavity. The initial clinical target volume (CTV) will be the GTV plus a margin of $2 \mathrm{~cm}$. If no surrounding edema is present, the initial clinical target volume (CTV) should include the contrastenhancing lesion (and should include the surgical resection cavity) plus a $2 \mathrm{~cm}$ margin. The CTV margin may be reduced to $0.5 \mathrm{~cm}$ around natural barriers to tumor growth such as the skull, ventricles, falx etc. and also to allow sparing of the optic nerve/chiasm, if necessary. The initial planning target volume (PTV1) is an additional margin of $0.5 \mathrm{~cm}$, to account for variations in set-up and reproducibility. Reducing PTV margins to modify organ at risk (OAR) dose(s) is not generally permissible. However, OAR must be defined, along with a planning volume at risk (PRV) for each OAR. Each PRV will be its OAR plus $3 \mathrm{~mm}$.

\section{Boost target volume}

The boost gross tumor volume (GTV2) will be defined by the contrast-enhanced T1 abnormality on the post-operative MRI scan. This must also include the surgical cavity. It may be compromised slightly where tumor is $5 \mathrm{~mm}$ from structures such as optic apparatus, brain stem if necessary. The boost planning target volume (PTV2) is an additional margin of $0.5 \mathrm{~cm}$, account for variations in set-up and reproducibility.

\section{Dose guidelines}

The initial planning target volume will be treated to 50 Gy in 25 fractions. After $50 \mathrm{~Gy}$, the cone down or boost planning volume will be treated to a total of $60 \mathrm{~Gy}$ in 30 fractions, with five additional fractions of $2 \mathrm{~Gy}$ each in 3DCRT. In case of IMRT initial planning target volume will be treated to $50 \mathrm{~Gy}$ in 30 fractions at 1.65 Gy per fraction and boost planning target volume will be treated to a total dose of $60 \mathrm{~Gy}$ in 30 fractions at $2 \mathrm{~Gy}$ per fraction simultaneously. The inhomogeneity within the target volume was kept within $\pm 10 \%$ of the prescribed dose. The minimum dose to the target volume should be kept within $10 \%$ of the dose at the center of the volume. Target dose was specified such that at least $95 \%$ of the PTV shall receive $95 \%$ of the prescribed dose (Table 1).

\begin{tabular}{|l|l|l|}
\hline Target & Definition & Comments \\
\hline GTV & T1 abnormality +resection cavity & Subset of tumors will be heterogeneously enhancing or grade III without enhancement. In these cases CTV=GTV. \\
\hline CTV & $\begin{array}{l}\text { T2 or FLAIR abnormality }+2 \mathrm{~cm} \\
+ \text { GTV }\end{array}$ & $\begin{array}{l}\text { Extension along pathways of spread such as corpus callosum may be considered, may be edited in areas where } \\
\text { tumor progression is not likely to occur (bone, sinus). }\end{array}$ \\
\hline PTV 1 & CTV $+0.5 \mathrm{~cm}$ & Account for variations in set-up and reproducibility (3-5 mm). \\
\hline PTV 2 & GTV $+0.5 \mathrm{~cm}$ & May be compromised slightly where tumor is $5-10 \mathrm{~mm}$ from structures such as optic apparatus, brain stem \\
\hline
\end{tabular}

Table 1: Target Volume Definition for Malignant Glioma [8]. 


\section{Dose limitation to critical structures}

Organ at risk volumes are contoured such as lenses of both eyes, both retina, both optic nerves, the optic chiasm, and the brainstem. The following dose limitation was aimed for the above organs at risk. Retina $<45$ Gy, optic nerves $<54$ Gy, Optic Chiasm $<54$ Gy, brainstem $<54 \mathrm{~Gy}$, lenses as low as achievable.

\section{D treatment planning}

Use of Beams-eye-view (BEV) Treatment planning attempts to arrange the treatment fields so as to conform the high dose region to the target minimize dose to normal structures and constrain the doses to critical structures to below tolerance. The target volume and normal structures delineated on transverse axial CT slices can be represented by $3 \mathrm{D}$ wire frame or solid objects on a computer monitor. This $3 \mathrm{D}$ information is used to define the geometry of the radiation fields to optimize the dose distribution using the Beam's Eye View (BEV) concept. The BEV display shows the anatomy from the perspective of a viewer positioned at the beam's source, looking at the patient along the central axis of the beam. Using a control panel the planner can adjust beam parameters, such as jaw positions, gantry, collimator and couch angles and the adjustments are reflected in the BEV display. To assist the planner to understand this $3 \mathrm{D}$ information, the intersection of the beams with transverse, sagittal and coronal planes are simultaneously displayed. The selection of beam parameters is based on these geometric considerations, planner's experience and some anatomical sites. For each planned beam direction the appropriate apertures can be designed either manually by outlining in BEV or automatically by specifying a margin around the target volume. The margin for beam penumbra is generally $0.5 \mathrm{~cm}$ to the periphery and $0.7 \mathrm{~cm}$ to the superior and inferior borders of the projected target volume in the BEV plane. The irregularly shaped beam apertures can be delivered using multi-leaf collimators (MLCs).

\section{D dose calculations}

Dose calculation algorithms for computerized treatment planning have been evolving since the middle of the 1950s. In broad terms the algorithms fall into three categories (a) correction-based, (b) modelbased, and (c) direct Monte Carlo. Either one of the methods can be used for 3-D treatment planning, although with a varying degree of accuracy and speed. However, the model-based algorithms and the direct Monte Carlo are becoming more and more the algorithms of the future. This is because of their ability to simulate radiation transport in three dimensions and therefore, more accurately predict dose distribution under conditions of charged particle disequilibrium, which can occur in low-density tissues such as lung and heterogeneous tissue interfaces. Although currently they are plagued by slow speed, this limitation is fast disappearing with the ever-increasing speed and data storage capacity of modern computers.

\section{Qualitative plan evaluation}

Traditionally treatment plans are optimized iteratively by using multiple fields, beam modifiers (e.g., wedges and compensators, etc.), beam weights, and appropriate beam directions. Dose distributions of competing plans are evaluated by viewing isodose curves in individual slices, orthogonal planes (e.g., transverse, sagittal, and coronal), or 3D isodose surfaces. The latter represent surfaces of a designated dose value covering a volume. An isodose surface can be rotated to assess volumetric dose coverage from different angles. The isodose curves displayed in orthogonal planes and an isodose surface just covering the target volume. One of the major advantages of $3 \mathrm{D}$ treatment planning is the display of dose distribution, which can be manipulated with ease to show volumetric dose coverage in individual slices, orthogonal planes, or as $3 \mathrm{D}$ isodose surfaces.

\section{Quantitative plan evaluation}

Display of dose distribution in the form of isodose curves or surfaces is useful not only because it shows regions of uniform dose, high dose, or low dose but also their anatomic location and extent. In $3 \mathrm{D}$ treatment planning, this information is essential but should be supplemented by dose-volume histograms (DVH) for the segmented structures, for example, targets, critical structures, etc. A DVH not only provides quantitative information with regard to how much dose is absorbed in how much volume but also summarizes the entire dose distribution into a single curve for each anatomic structure of interest. It is, therefore, a great tool for evaluating a given plan or comparing competing plans.

The DVH may be represented in two forms: the cumulative integral DVH and the differential DVH. The cumulative DVH is a plot of the volume of a given structure receiving a certain dose or higher as a function of dose. Any point on the cumulative DVH curve shows the volume that receives the indicated dose or higher. The differential DVH is a plot of volume receiving a dose within a specified dose interval (or dose bin) as a function of dose. The differential form of DVH shows the extent of dose variation within a given structure. For example, the differential DVH of a uniformly irradiated structure is a single bar of $100 \%$ volume at the stated dose. Of the two forms of DVH, the cumulative DVH has been found to be more useful and is more commonly used than the differential form.

\section{Comparative evaluation of treatment plans}

In this study, dosimetric analysis of 3DCRT and IMRT plans was performed for each of the 20 patients by both qualitative and quantitative measures. Isodose distribution was first compared visually on axial, sagittal and coronal slices for degree of conformity of the prescribed dose to the PTV and then for any inclusion of OAR within high dose and low dose levels. Specifically, we examined isodose lines from $5 \mathrm{~Gy}$ and up in our evaluation. Direct comparison was also made of the cumulative DVH curves for PTV, OAR, and non-target tissue. Integral dose to non-target brain tissue (Brain-PTV) was evaluated. Plan comparison was also made quantitatively by comparing DVH parameters and by computing and comparing relevant metrics for target coverage, target conformity, dose heterogeneity within the target, and critical normal tissue sparing. Target coverage was assessed by comparing the minimum and maximum doses to PTV $\left(\mathrm{D}_{\min }\right.$ and $\mathrm{D}_{\max }$ respectively) and the percentage of PTV covered by at least $90 \%$, $95 \%, 100 \%$ of the prescribed dose $\left(\mathrm{V}_{90 \%}, \mathrm{~V}_{95 \%}, \mathrm{~V}_{100 \%}\right.$ respectively $)$. Homogeneity of dose within the target volume has been assessed previously by using an in homogeneity coefficient (IC) as defined by

$$
\mathrm{IC}=\left(\mathrm{D}_{5 \%}-\mathrm{D}_{95 \%}\right) / \mathrm{D}_{\text {mean }}
$$

Where $\mathrm{D}_{5 \%}$ and $\mathrm{D}_{95 \%}$ represent the dose levels on the DVH curve corresponding to $5 \%$ and $95 \%$ of the target volume, respectively, and Dmean represents the mean dose to the target. Ideally this index value should be equal to zero for uniformity of the target. Conformity of high doses around the target has been evaluated by calculating the conformity index (CI) at a given isodose level (e.g. CI 95\%), as defined 
Citation: Khaleel IA, Govardhan HB, Senthil K (2018) Comparison of Intensity Modulated Radiation Therapy (IMRT) and Three Dimensional Conformal Radiotherapy (3DCRT) In Supratentorial Astrocytic Series WHO Grade III-IV Primary Malignant Brain Tumors. J Nucl Med Radiat Ther 9: 368. doi:10.4172/2155-9619.1000368

Page 4 of 7

by the ratio of the volume of total tissue receiving at least $95 \%$ of the prescribed dose to the volume of PTV such that

Conformity Index=Volume within the 95\% isodose/volume of PTV

Which is ideally should be unity. Although the CI does not account for the relationship of the PTV to the $95 \%$ isodose volume, it is assumed that the PTV is included within the $95 \%$ volume based on our planning criteria which require that at least $95 \%$ (in reality $96 \%-98 \%$ is usually achieved ) of the PTV receives the prescribed dose. Integral dose to normal brain tissue (Brain-PTV) was calculated by multiplying the volume of the Brain PTV and mean dose to the structure volume.

\section{Statistical analysis}

Analysis were performed by using a paired two-tailed student $t$ test to determine if there was a significant differences in any of the parameters examined. Differences were considered statistically significant at $\mathrm{p} \leq 0.05$.

\section{Results}

\section{Patient characteristics}

Sixty patients of histologicaly and radiologicaly proved malignant glioma, having post-operative KPS scale of 70, and above were received Radiotherapy at Kidwai memorial institute of oncology. Patient characteristic are shown in Table 2. There were 48 males and 12 females in the study group with median age of 44 years (25-60 years). Glioblastoma accounts for the $80 \%$ of the cases, remaining $20 \%$ were anaplastic astrocytoma histology. The average duration between surgery and start of radiotherapy is 5 weeks, ranged from 3 weeks to 7 weeks. Our study included tumor sizes which ranged from $239 \mathrm{~cm}^{3}$ to $671 \mathrm{~cm}^{3}$ for initial PTV and $32 \mathrm{~cm}^{3}$ to $386 \mathrm{~cm}^{3}$ for Boost PTV.

\begin{tabular}{|c|c|}
\hline \multicolumn{2}{|c|}{ Gender } \\
\hline Male & 48 \\
\hline Female & 12 \\
\hline \multicolumn{2}{|c|}{ Age (year) } \\
\hline Median & 44 \\
\hline Range & $25-60$ \\
\hline WHO Grade III & 12 \\
\hline WHO Grade IV & 48 \\
\hline \multicolumn{2}{|c|}{ Tumor Location } \\
\hline Temporal & 6 \\
\hline Parietal & 9 \\
\hline Frontal & 12 \\
\hline Combined & 33 \\
\hline \multicolumn{2}{|c|}{ Side } \\
\hline Right & 24 \\
\hline Left & 33 \\
\hline Crossing Midline & 9 \\
\hline
\end{tabular}

\begin{tabular}{|l|l|}
\hline \multicolumn{2}{|c|}{ Surgical Treatment } \\
\hline Gross Total Resection & 27 \\
\hline Sub Total Resection & 21 \\
\hline Biopsy & 12 \\
\hline \multicolumn{2}{|c|}{ Performance Status } \\
\hline KPS 90 & 9 \\
\hline KPS 80 & 33 \\
\hline KPS 70 & 18 \\
\hline \multicolumn{1}{|c|}{ Duration between surgery and start of RT } \\
\hline Average & 5 weeks \\
\hline Range & $3-7$ weeks $^{\prime}$ \\
\hline \multicolumn{2}{|c|}{ Target } \\
\hline Initial PTV1 (mean) & $358 \mathrm{cc}^{3}$ \\
\hline Range & $239-671 \mathrm{cc}^{3}$ \\
\hline Boost PTV2 (mean) & $126 \mathrm{cc}^{3}$ \\
\hline Range & $32-386 \mathrm{cc}^{3}$ \\
\hline
\end{tabular}

Table 2: showing patients characteristics.

\section{Isodose distribution and DVH analysis}

Intensity modulated radiotherapy plans used five to seven fields when compared to 3DCRT plans used 2 to 4 fields. In this study $6 \mathrm{MV}$ photon is used for all beams. Representative 3DCRT and IMRT plans for 2 patients are shown in Figures 1 and 2. The plan in Figure 3 was generated for patient with post-operative malignant glioma in the right parietal lobe with volume of Boost PTV $=89.5 \mathrm{~cm}^{3}$, PTV $50=295.6 \mathrm{~cm}^{3}$ Isodose distribution for a 3DCRT plan with two wedged beams and IMRT plan with 5 fields are shown in Figure 3.

The Prescribed isodose volume in all dimensions examined with IMRT (transverse slice shown) compared with 3DCRT. However careful inspection of the low dose volumes from 5 to 30 Gy clearly shows that IMRT also restricts spread of low doses, especially at $20 \mathrm{~Gy}$ and $10 \mathrm{~Gy}$, to the optic chiasm, Brain stem and Lt Eye in this patient. DVH comparisons are shown in Figure 3.

Though the $\mathrm{D}_{\max }$ of the Boost PTV decreased slightly from $62.95 \mathrm{~Gy}$ to $62.47 \mathrm{~Gy}$, the target conformity index improved substantially, with Conformity index (CI) for the Boost PTV decreasing from 2.6 to 1.16 for 3DCRT compared to IMRT plan. The DVH for Optic Chiasm and Brainstem to a lesser extent was improved with IMRT which reduced $\mathrm{D}_{\max }$ for Optic Chiasm from 35.8 Gy to $21.45 \mathrm{~Gy}$ and Mean dose from 27.2 to $16.29 \mathrm{~Gy}$, for Brain Stem the $\mathrm{D}_{\max }$ reduced from $57.53 \mathrm{~Gy}$ to $48.50 \mathrm{~Gy}$ and Mean dose from $43.59 \mathrm{~Gy}$ to $29.07 \mathrm{~Gy}$. The integral dose to Brain-PTV volume was $25.36 \mathrm{~Gy} \mathrm{~cm}^{3}$ for 3DCRT plan and $24.14 \mathrm{~Gy}$ $\mathrm{cm}^{3}$ for IMRT. 
Citation: Khaleel IA, Govardhan HB, Senthil K (2018) Comparison of Intensity Modulated Radiation Therapy (IMRT) and Three Dimensional Conformal Radiotherapy (3DCRT) In Supratentorial Astrocytic Series WHO Grade III-IV Primary Malignant Brain Tumors. J Nucl Med Radiat Ther 9: 368. doi:10.4172/2155-9619.1000368

Page 5 of 7
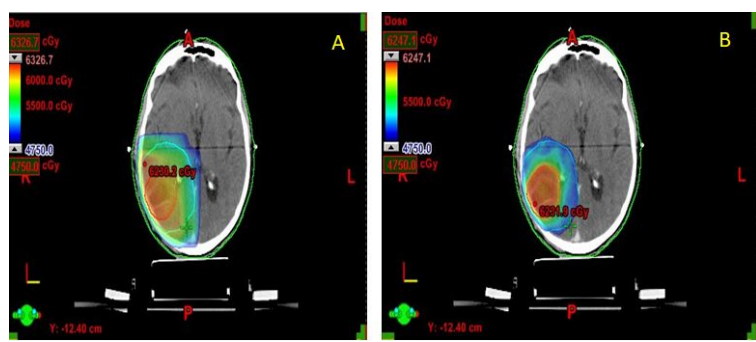

Figure 1: Isodose distribution of patient Rt. parieto-occipital glioma planned with a) 3DCRT b) IMRT.
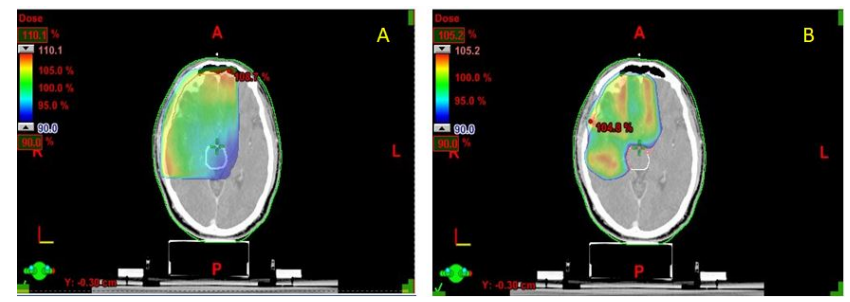

Figure 2: Isodose distribution of patient Rt. Parieto-occipital glioma planned with a) 3DCRT b) IMRT.

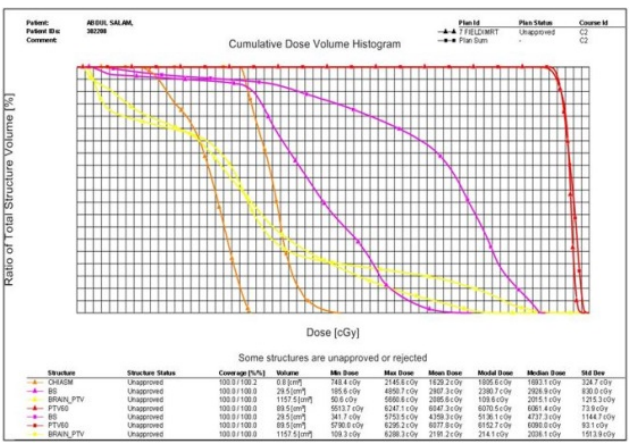

Figure 3: Cumulative dose volume histogram of patient with postoperative malignant glioma in the right parietal lobe glioma. A) 3DCRT B) IMRT.

The $\mathrm{D}_{\text {mean }}$ of the brain stem, optic chiasm and Brain-PTV with IMRT was less by $25 \%, 66 \%, 13 \%$ compared with 3DCRT. The conformity index (CI) of the initial PTV improved from 1.6 to 1.15 and boost PTV from 1.93 to 1.19 with IMRT technique and the DVH revealed no increase in normal tissue dose (Brain-PTV). Quantitative assessment showed that IMRT reduced the total non-target brain tissue integral dose from $24.86 \mathrm{~Gy} . \mathrm{cm}^{3}$ to $21.60 \mathrm{~Gy} . \mathrm{cm}^{3}$ (Approximately $13 \%)$.

\section{Target coverage}

A quantitative analysis was performed in aggregate for the 120 plans (60 IMRT plans and 60 3DCRT plans) using DVH data and comparing mean values for the inhomogeneity coefficient (IC) and the target coverage parameters $\mathrm{D}_{\min }, \mathrm{D}_{\max }, \mathrm{V}_{100}, \mathrm{~V}_{95}$, and $\mathrm{V}_{90}$ for the PTV and values are shown in Table 3. Both initial and Boost PTV were analyzed and showed no significant difference between 3DCRT and IMRT plans in terms of the mentioned parameters except minimum and maximum dose. In boost PTV, $\mathrm{D}_{\max }$ dose reduced from $62.88 \mathrm{~Gy}$ to $62.11 \mathrm{~Gy}$ $(\mathrm{p}=0.0037)$ and $\mathrm{D}_{\min }$ dose reduced from $57.15 \mathrm{~Gy}$ to $54.21 \mathrm{~Gy}$ $(\mathrm{p}<0.0001)$ for IMRT technique. In initial PTV (PTV 50), the $\mathrm{D}_{\max }$ was reduced from $63.30 \mathrm{~Gy}$ to $61.72 \mathrm{~Gy},(\mathrm{p}<0.0133)$ and $\mathrm{D}_{\min }$ was reduced from $43.65 \mathrm{~Gy}$ to $39.11 \mathrm{~Gy}(\mathrm{p}<0.0003)$. 3DCRT and IMRT plans both achieved comparably excellent target coverage with $90 \%-95 \%$ of PTV receiving more than 99 to $100 \%$ of prescribed dose (Figures 1 and 2). Dose uniformity within the target volumes were almost similar and indicated high degree of dose uniformity. Dose uniformity in PTV 50 volume was almost similar between 3DCRT and IMRT plans. In Boost PTV, the dose uniformity was slightly better for IMRT compared to 3CDRT plan (Table 3).

\begin{tabular}{|c|c|c|c|c|}
\hline & 3DCRT (Gy or \%) & IMRT (Gy or \%) & $\%$ diff & $\mathbf{p}$ \\
\hline \multicolumn{5}{|c|}{ PTV 50} \\
\hline$D_{\min }$ & $43.65 \pm 6.20$ & $39.11 \pm 5.10$ & -10.4 & $0.0133^{*}$ \\
\hline$D_{\max }$ & $63.30 \pm 1.04$ & $61.72 \pm 1.09$ & -2.49 & $0.0003^{*}$ \\
\hline$V_{90 \%}$ & $99.75 \pm 0.59$ & $99.85 \pm 0.20$ & 0.1 & 0.5106 \\
\hline$V_{95 \%}$ & $99.13 \pm 2.06$ & $99.10 \pm 0.65$ & -0.03 & 0.9572 \\
\hline IC & $0.164 \pm 0.03$ & $0.177 \pm 0.2$ & 7.9 & 0.2383 \\
\hline $\mathrm{Cl}$ & $1.81 \pm 0.3$ & $1.14 \pm 0.06$ & -37 & $<0.0001$ \\
\hline \multicolumn{5}{|c|}{ PTV 60} \\
\hline$D_{\min }$ & $57.15 \pm 1.20$ & $54.21 \pm 2.47$ & -5.14 & $0.0001^{*}$ \\
\hline $\mathrm{D}_{\max }$ & $62.88 \pm 1.02$ & $62.11 \pm 0.82$ & -1.22 & $0.0037^{*}$ \\
\hline$V_{90 \%}$ & $100.0 \pm 0.00$ & $100.0 \pm 0.00$ & 0 & NS \\
\hline$V_{95 \%}$ & $99.90 \pm 0.25$ & $99.65 \pm 0.43$ & -0.25 & 0.122 \\
\hline$V_{100 \%}$ & $67.03 \pm 14.0$ & $73.24 \pm 18.3$ & 9.26 & 0.2321 \\
\hline IC & $0.06 \pm 0.02$ & $0.04 \pm 0.01$ & -33 & $0.0015^{\star}$ \\
\hline $\mathrm{Cl}$ & $3.04 \pm 0.8$ & $1.17 \pm 0.06$ & -61 & $<0.0001$ \\
\hline
\end{tabular}

Table 3: Target coverage for PTV 50 and PTV 60 volumes.

\section{Target conformity and normal tissue sparing}

IMRT achieved a significant improvement in target conformity in both initial PTV and boost PTV volumes in all patients Tables 3 and 4 . In initial PTV volume, there was significant difference between two plans. Conformity index was improved from 1.81 in 3DCRT to 1.14 in IMRT with approximate difference of $30 \%$ with $\mathrm{p}<0.0001$. Conformity around the boost PTV volume was improved in all 60 patients in IMRT on an average by $60 \%$ compared to 3DCRT. It was improved from 3.04 to $1.17(\mathrm{p}<0.0001)$. Better conformity in high dose volumes was correlated with substantial reduction in both mean dose and maximum dose to the examined critical normal tissues.

All the normal structures examined showed significant statistical differences $(p<0.05)$ between IMRT and 3CDRT plans. The maximum dose to Brain stem, Optic chiasm, Lt optic nerve, Rt optic nerve, Lt eye 
Citation: Khaleel IA, Govardhan HB, Senthil K (2018) Comparison of Intensity Modulated Radiation Therapy (IMRT) and Three Dimensional Conformal Radiotherapy (3DCRT) In Supratentorial Astrocytic Series WHO Grade III-IV Primary Malignant Brain Tumors. J Nucl Med Radiat Ther 9: 368. doi:10.4172/2155-9619.1000368

Page 6 of 7

and Rt eye was decreased by $12 \%, 22 \%, 38 \%, 46 \%, 35 \%, 29 \%$ respectively, with IMRT (Table 4) compared to 3DCRT technique. The mean doses to these structures were decreased by $17 \%, 33 \%, 42 \%, 54$ $\%$, 33\%, 37\% respectively, with IMRT. Dose received by $50 \%$ of the volume of the normal brain tissues $\left(\mathrm{D}_{50}\right)$ were analyzed and showed improvement in IMRT plans compared with 3DCRT plan. D50 to these structures were decreased by $5.6 \%, 33.9 \%, 43.4 \%, 56.44 \%$, $37.69 \%$, and $34.7 \%$ respectively for IMRT plans. In cases where the normal tissue tolerances were exceeded by 3DCRT, in such cases IMRT allowed the $\mathrm{D}_{\max }$ dose to be lowered to below the tolerance dose of the normal tissues, where the PTV directly abuts the brain stem or optic chiasm and a compromise was made to ensure adequate coverage of the PTV volumes.

\begin{tabular}{|c|c|c|c|c|}
\hline & 3DCRT (Gy) & IMRT (GY) & $\%$ diff & $\mathbf{p}$ \\
\hline \multicolumn{5}{|c|}{ Brain Stem } \\
\hline$D_{\text {mean }}$ & $25.35 \pm 9.87$ & $20.97 \pm 10.5$ & -17 & $0.0040^{*}$ \\
\hline$D_{\max }$ & $52.64 \pm 9.63$ & $46.22 \pm 9.86$ & -12.19 & $<0.0001^{*}$ \\
\hline $\mathrm{D}_{50}$ & $17.38 \pm 15.5$ & $16.4 \pm 13.70$ & -5.63 & 0.4716 \\
\hline \multicolumn{5}{|c|}{ Optic Chiasm } \\
\hline$D_{\text {mean }}$ & $26.83 \pm 17.2$ & $17.72 \pm 11.7$ & -33.95 & $0.0011^{*}$ \\
\hline$D_{\max }$ & $37.03 \pm 22.0$ & $26.58 \pm 16.0$ & -22.8 & $0.0003^{*}$ \\
\hline $\mathrm{D}_{50}$ & $26.45 \pm 17.6$ & $17.48 \pm 12.0$ & -33.91 & $0.0016^{*}$ \\
\hline \multicolumn{5}{|c|}{ Left Optic Nerve } \\
\hline $\mathrm{D}_{\text {mean }}$ & $18.4 \pm 17.63$ & $10.60 \pm 10.6$ & -42.31 & $0.0023^{*}$ \\
\hline$D_{\max }$ & $26.5 \pm 20.90$ & $16.36 \pm 14.9$ & -38.64 & $0.0008^{*}$ \\
\hline $\mathrm{D}_{50}$ & $18.4 \pm 18.20$ & $10.40 \pm 10.5$ & -43.4 & $0.0030^{*}$ \\
\hline \multicolumn{5}{|c|}{ Right Optic Nerve } \\
\hline$D_{\text {mean }}$ & $19.77 \pm 18.6$ & $8.93 \pm 7.390$ & -54.83 & $0.0020^{*}$ \\
\hline$D_{\max }$ & $25.17 \pm 20.5$ & $13.41 \pm 10.2$ & -46.72 & $0.0012^{*}$ \\
\hline$D_{50}$ & $20.16 \pm 19.2$ & $8.78 \pm 7.39$ & -56.44 & $0.0021^{*}$ \\
\hline \multicolumn{5}{|c|}{ Right Eye } \\
\hline$D_{\text {mean }}$ & $12.18 \pm 13.7$ & $7.65 \pm 7.49$ & -37.19 & 0.0067 \\
\hline$D_{\max }$ & $20.02 \pm 17.7$ & $14.2 \pm 11.2$ & -29.07 & $0.009^{*}$ \\
\hline $\mathrm{D}_{50}$ & $11.64 \pm 14.2$ & $7.60 \pm 7.62$ & -34.7 & $0.0168^{*}$ \\
\hline \multicolumn{5}{|c|}{ Left Eye } \\
\hline$D_{\text {mean }}$ & $8.95 \pm 10.6$ & $5.92 \pm 5.65$ & -33.85 & $0.0439^{*}$ \\
\hline$D_{\max }$ & $19.26 \pm 17.8$ & $12.4 \pm 8.71$ & -35.16 & $0.0085^{*}$ \\
\hline $\mathrm{D}_{50}$ & $8.17 \pm 11.10$ & $5.09 \pm 5.22$ & -37.69 & $0.0461^{*}$ \\
\hline Brain-PTV (Mean) & $23.88 \pm 4.6$ & $21.79 \pm 3.65$ & -8.75 & $0.0003^{*}$ \\
\hline
\end{tabular}

Table 4: Dosimetric variation between IMRT and 3DCRT to critical normal structure.

\section{Integral dose to normal brain tissue}

Evaluation of integral dose (ID) of normal brain tissue showed that there was no increase in the integral dose to Brain-PTV structure examined in IMRT compared with 3DCRT plans Table 4. IMRT plans decreased the integral dose to normal brain tissue by approximately $8 \%,(\mathrm{p}<0.0003)$ compared to 3DCRT. When comparing the integral dose of individual patients out of 60 patients, $90 \%$ of the patients had absolute reduction of integral dose with IMRT and only about $10 \%$ of patient showed high integral dose.

\section{Discussion}

There has been a dramatic improvement in radiotherapy techniques over the last two decades. Improvements in dose distribution and local control have been observed with 3DCRT as compared with conventional two dimensional treatment planning. It has also been showed that the morbidity of therapy decreased with the use of 3DCRT compared with conventional treatment planning. Furthermore, IMRT has shown improvement in target dose conformity, as well as reduction in the dose to the normal tissues while achieving comparable target coverage when compared with 3DCRT techniques in many treatment sites including oesophgous, prostate, paranasal sinuses, nasopharynx and other head and neck sites $[3,4,6,8-11]$.

In case of treatment of malignant glioma with standard therapy consisting of maximal safe surgical resection followed by involved field radiation therapy and chemotherapy has shown survival advantage in favourable prognostic groups. Uncertainties in target volume definition may not only result in marginal misses of tumor but also in unnecessarily overdosing the normal brain. The recent developments in CNS imaging technology like CT and MRI fusion in radiotherapy planning and functional imaging may further increases the ability to more precisely define the target volume and target the areas at risk of failure. If gliomas can accurately mapped, IMRT may provide further advantage because of its ability to target selected more resistance parts within the tumor with higher radiation doses without increasing the dose to normal tissue. As the number of long term survivors increases, an increase will almost certainly be seen in the number of patients suffering from the late effect of radiation. Therefore to ensure optimal coverage with minimal radiation injury, investigating the integration of advanced, highly conformal radiotherapy techniques for this disease is important. This study was a comparative dosimetric evaluation of IMRT and 3DCRT for treatment of 60 patients of malignant glioma, with respect to target coverage, conformity of prescribed dose volume, sparing of organ at risk and integral dose to non-target normal brain tissue.

Comparison of IMRT and 3DCRT for the malignant glioma of the brain are scarce in literature [5,12]. Chan et al. with a study, group of 5 patients demonstrated that, simultaneous boost in IMRT delivered higher dose to the gross tumor volume while respecting same critical normal tissue constraint and also still maintaining the uninvolved normal brain tissue at dose levels of the 3DCRT [13,14]. One more study by Narayana et al. analyzed 20 patients, showed that regardless of tumor location IMRT did not lead to significant improvement in target coverage (maximum dose, minimum dose ,or D95 coverage) when compared to 3DCRT [13]. Our dosimetric analysis confirmed that there was no significant difference in target coverage between IMRT and 3DCRT plans with slight superiority in 3DCRT plan in the range of $95 \%-100 \%$ of prescribed dose. Both techniques were shown good 
Citation: Khaleel IA, Govardhan HB, Senthil K (2018) Comparison of Intensity Modulated Radiation Therapy (IMRT) and Three Dimensional Conformal Radiotherapy (3DCRT) In Supratentorial Astrocytic Series WHO Grade III-IV Primary Malignant Brain Tumors. J Nucl Med Radiat Ther 9: 368. doi:10.4172/2155-9619.1000368

Page 7 of 7

target coverage in initial PTV and boost PTV. For many gliomas target coverage and dose uniformity are excellent with standard 3DCRT techniques owing to the nearly spherical or cylindrical shape of the lesion. Therefore it was not surprising that significant further improvement was not observed with IMRT. Target coverage and dose uniformity improvement with IMRT have been primarily reported in sights like Head and Neck or Prostate [8,9], where the target is concave, surrounding normal tissues with dose limits much less than that of the tumor. Gliomas can be highly irregular but typically exhibit few concavities. When concavities do exist such as when the tumors surrounds the chiasm the required dose gradient between tumor and normal tissues is often less than that observed in other sites. As a result very good target coverage is often achieved with $3 \mathrm{D}$ planning. However as we escalate the prescription dose for this tumors even if only to areas of suspected high tumor density, the benefit of IMRT might increase because steeper dose gradients and more concave dose distributions will be necessary. Our study showed almost similar dose uniformity within the target volume both in 3DCRT and IMRT as indicated by high degree of dose uniformity.

Our data are comparable to those reported by Hermanto et al. where IMRT did not further improve target coverage or dose uniformity within the target, but it did results in statistically significant superiority in target conformity $(\mathrm{p}<0.001)$, and also significant reduction in the mean and maximum doses to the critical structures like brain stem , optic pathway $(\mathrm{p}<0.05)$ [7]. In IMRT if the normal structures like eloquent cortex, brain stem and optic pathway is located near the target, there is actually a compromise to be done in normal tissue sparing and target coverage in the range of 95\%-100\% of prescribed dose, because if we optimize stringent dose constraint for normal tissue located nearby target it was trying to create cold spot within the target. Dose received by the $50 \%$ of the volume of critical normal tissue was improved in IMRT plans compared to 3DCRT plan.

The integral dose was evaluated for Brain-PTV, the average normal brain tissue integral dose was reduced in IMRT compared with 3DCRT by approximately $8 \%$. These finding are comparable with majority of the published studies. A study by Hermanto et al. [7], demonstrated IMRT decreased the total integral dose to the non-target brain tissue by $7 \%-10 \%$, Narayana et al. [14], reported a $7 \%$ decrease in mean dose to normal brain with IMRT compared with 3DCRT. In our study, 90\% of the patients had absolute reduction of integral dose with IMRT and only about $10 \%$ of patient showed high integral dose. The reason for this could be in those cases the tumor was located eccentrically in the occipital lobe and this was adequately covered with two fields with 3DCRT techniques, whereas for the treatment of the same target with IMRT multiple fields at different angulations need to be selected. The passage of beams through larger depth might tend to increase the integral dose to non-target brain. It together underscores the fact that with careful IMRT planning integral dose to the normal tissues can be significantly decreased.
With careful planning in regard to choice of beam angles, beam weighting, and recognition of potential exposure of normal tissues to exit dose, our study showed that IMRT enabled improvement in target dose conformity, critical tissue sparing, and reduction of integral dose. This superior dosimetric advantage of IMRT may prove useful in reducing dose to the surrounding critical structures when tumor is situated very close to these structures, in minimizing the treatment related morbidity like cognition deficit, to improve quality of life and also may have an option to re-irradiate for recurrence of tumor when indicated in long time survivors.

\section{References}

1. Deacon JM, Peckman MJ, Steel G (1984) The radio responsiveness of human tumors and the initial slope of the cell survival curve. Rad Oncol 2: 317-323.

2. Webb S (1998) Advances in treatment with intensity modulated conformal radiotherapy. Tumori 84: 112-126.

3. Hunt MA, Zelefsky MJ, Wolden S (2001) Treatment planning and delivery of intensity modulated radiation therapy for primary nasopharynx cancer. Int J Radiat Oncol Biol Phys 49: 623-632.

4. Catharine HC, Margaret B, Cephas DM (2004) Intensity modulated radiotherapy improves target coverage, spinal cord sparing and allows dose escalation in patients with locally advanced cancer of the larynx. Int J Radiate Onc Biol Phys 70: 189-198.

5. Shannom MM, Shalahuddin A, Stefanos K (2007) Intensity modulated radiotherapy vs three dimensional conformal radiotherapy for treatment of high grade glioma. J App clin Med Phys 8: 47-60.

6. Andrea P, Mark C, Frank L (2000) Comparison of intensity modulated radiotherapy with conventional conformal radiotherapy for complex shaped tumors. Int J Rad Unclog Biol Phys 48: 1371-1380.

7. Ulrich H, Erik KF, Ming FJ (2007) Does IMRT increase the integral dose the normal brain?. Int J Rad Oncol Biol Phys 67: 1135-1144.

8. Edwin PMJ, Luc GHD, Marcel VH, Harry B (2000) Target volume in radiotherapy for high grade glioma of brain. Rad Oncol 56: 151-156.

9. Zelefsky MJ, Fuks Z, Hunt M (2002) High dose intensity modulated radiation therapy for prostate cancer. Int J Radiate Unclog Biol Phys 53: 1111-1116.

10. Nutting CM, Bedford JL, Cosgrove VP (2001) A comparison of conformal and intensity modulated techniques for esophageal radiotherapy. Rad Oncol 61: 157-163.

11. Breen SL, Kehagioglou P, Usher C (2004) A comparison of conventional, conformal and intensity modulated coplanar radiotherapy plans for posterior fossa treatment. Br J Radiol 77: 768-774.

12. Ashwatha N, Josh Y, Sean B (2006) Intensity modulated radiotherapy in high grade glioma: Clinical and dosimetric results. Int J Radiate Unclog Biol Phys 64: 892-897.

13. Maria FC, Karen S, Chandra B (2003) Comparison of intensity modulated radiotherapy with three dimensional conformal radiation therapy planning for glioblastoma multiforme. Med Dos 28: 261-165.

14. Brahme A (1988) Optimization of stationary and moving beam radiation therapy Techniques. Rad Oncol 12: 129-140 